\title{
Universal quantum signature of mixed dynamics in antidot lattices
}

\author{
J. P. Keating, ${ }^{1}$ S. D. Prado, ${ }^{2}$ and M. Sieber ${ }^{1}$ \\ ${ }^{1}$ School of Mathematics, University of Bristol, Bristol BS8 1TW, United Kingdom \\ ${ }^{2}$ Instituto de Física, Universidade Federal do Rio Grande do Sul, 91501-970 Porto Alegre, RS, Brazil
}

(Received 9 September 2005; published 28 December 2005)

\begin{abstract}
We investigate phase coherent ballistic transport through antidot lattices in the generic case where the classical phase space has both regular and chaotic components. It is shown that the conductivity fluctuations have a non-Gaussian distribution, and that their moments have a power-law dependence on a semiclassical parameter, with fractional exponents. These exponents are obtained from bifurcating periodic orbits in the semiclassical approximation. They are universal in situations where sufficiently long orbits contribute.
\end{abstract}

DOI: 10.1103/PhysRevB.72.245334

PACS number(s): 73.23.Ad, 75.47.Jn, 05.45.Mt, 03.65.Sq

\section{INTRODUCTION}

Experiments on mesoscopic semiconductor devices have exhibited a variety of features that can be attributed to quantum chaos. ${ }^{1-3}$ Modern fabrication techniques make it possible to produce extremely pure semiconductor microstructures in which the motion of the electrons is confined to two-dimensional domains whose relevant dimensions are much smaller than the phase coherence length and the transport mean-free-path. In this ballistic regime semiclassical methods have been very successful in connecting quantum interference effects to the underlying classical dynamics. ${ }^{4,5}$

There has been a considerable emphasis on systems in which the confinement potential leads to chaotic motion. As in disordered systems, certain transport properties are found to be universal, like conductance fluctuations and weak localization properties of transport through cavities. ${ }^{6-11}$ In contrast, if the dynamics is integrable, these features are in general not universal but depend on the specific system. Semiclassical methods have been applied to explain universal properties of chaotic transport as well as the nongeneric behavior of integrable cavities. ${ }^{6,7,9,12,13}$

Other experiments have been concerned with revealing signatures of classical periodic orbits in quantum phenomena. Examples are orbital magnetism in ballistic microstructures ${ }^{14}$ and transport through antidot superlattices. ${ }^{15-19}$ The latter consists of a two-dimensional electron gas at the interface of a GaAs $/ \mathrm{Al}_{x} \mathrm{Ga}_{1-x} \mathrm{As}$ heterostructure into which a periodic array of holes is drilled. The effective potential is a periodic structure of high potential peaks, and if the potential is steep it may be considered as an experimental realization of the Sinai billiard. Experiments on ballistic transport at temperature $T \approx 1.5 \mathrm{~K}$ show a series of pronounced peaks in the longitudinal conductivity vs magnetic field that can be explained by classical electron transport within the Drude formalism. ${ }^{15,16}$ Experiments at a lower temperature $T \approx 0.4 \mathrm{~K}$ reveal additional quantum oscillations superimposed on the classical peaks. ${ }^{17}$ These quantum oscillations can be attributed to unstable periodic orbits of the electrons in the confinement potential by a semiclassical theory for the conductivity. ${ }^{18,19}$

Generic systems have a phase space which is neither completely chaotic nor integrable but contains a mixture of regular islands and chaotic regions. This is relevant for experi- ments because confinement potentials are not hard-wall potentials, and soft-wall potentials typically lead to mixed dynamics. A natural question is whether ballistic mesoscopic systems have characteristic properties that differ from those of chaotic and integrable systems. One signature of mixed dynamics has been found in the ballistic transport through cavities. It was predicted and observed experimentally that the variance of conductance fluctuations, as a function of the magnetic field, has a power-law dependence with a noninteger exponent that is related to the trapping of chaotic trajectories near regular islands. ${ }^{20,21}$ The purpose of this paper is to show that there is a different mechanism in ballistic transport through antidot lattices which leads to universal behavior in mixed systems.

Amongst the main characteristics of the dynamics in mixed systems are bifurcations of periodic orbits, events in which different periodic orbits coalesce when parameters of the system are varied. Bifurcations are important for semiclassical approximations because bifurcating orbits carry a semiclassical weight that is higher than that of unstable periodic orbits and sometimes even that of tori of regular orbits. The dominating influence of bifurcations on transport through antidot lattices has been demonstrated in Refs. 22 and 23. Bifurcations occur in different forms, and depending on the physical quantity that one considers, they are of different importance. In the following we consider moments of conductivity fluctuations. We show that they are dominated by a competition between different types of bifurcations, leading to a power-law dependence on a semiclassical parameter with fractional exponents. These exponents are universal if the competition is amongst all generic bifurcations. Similar results have been obtained for moments of spectral counting functions and wave functions in closed mixed systems. ${ }^{24-27}$

In the following we briefly review the semiclassical theory of transport through antidot lattices and discuss modifications in the presence of bifurcations. We give an overview of different types of bifurcations and discuss their influence on moments of conductivity fluctuations.

\section{SEMICLASSICAL THEORY FOR THE CONDUCTIVITY}

The starting point for the semiclassical theory is the Kubo linear response theory. The conductivity is given in terms of 
matrix elements of the current operator. The semiclassical approximation of the matrix elements $\mathrm{s}^{28}$ and the application of the stationary phase method for evaluating integrals then yields a semiclassical expression for the conductivity as a sum over the classical (Drude) component ${ }^{16}$ and an oscillatory component in terms of periodic orbits. ${ }^{18,19}$

The Kubo formula for the longitudinal conductivity is ${ }^{29}$

$$
\sigma_{x x}=\frac{e^{2} \pi \hbar}{V} \operatorname{Tr}\left\{\hat{v}_{x} \delta_{\Gamma}\left(E_{F}-\hat{H}\right) \hat{v}_{x} \delta_{\Gamma}\left(E_{F}-\hat{H}\right)\right\},
$$

where $\hat{H}=(1 / 2 m)[\hat{\mathbf{p}}-e \hat{\mathbf{A}}(\hat{\mathbf{r}})]^{2}+U(\mathbf{r})$ is the Hamiltonian of a two-dimensional electron gas in a perpendicular magnetic field, $\mathbf{A}=(-B y / 2, B x / 2)$ is the vector potential taken in the symmetric gauge, $U(\mathbf{r})$ is the confinement potential, and $\hat{v}_{x}$ is the $x$ component of the velocity operator. $E_{F}$ denotes the Fermi energy, $m$ is the effective mass of the electron, and $V$ is the area of the system. Weak disorder is taken into account at the level of Born approximation by giving the $\delta$ functions a finite width $\Gamma=\hbar / 2 \tau_{e l}$ where $\tau_{e l}$ is the elastic scattering time. This is sufficient for our investigation of the influence of periodic orbits on the conductivity. Although a particular realization of the smooth disorder potential changes the classical trajectories slightly, this does not affect the following analysis of the generic bifurcations that they undergo if system parameters are changed.

To evaluate the Kubo formula (1) it is convenient to write the delta function in terms of retarded and advanced Green functions: $\quad \delta_{\Gamma}\left(E_{F}-\hat{H}\right)=-(1 / 2 \pi i)\left[\hat{G}^{+}\left(E_{F}\right)-\hat{G}^{-}\left(E_{F}\right)\right] \quad$ with $\hat{G}^{ \pm}(E)=[E-\hat{H} \pm i \Gamma]^{-1}$. In the semiclassical limit as $\hbar \rightarrow 0$, the longitudinal conductivity is reduced to terms that involve only products of the retarded and advanced Green functions, because terms that involve products of Green functions of the same type vanish: ${ }^{18,19}$

$$
\begin{aligned}
\sigma_{x x} \approx & 2 \frac{e^{2} \pi \hbar}{V}\left(\frac{1}{2 \pi}\right)^{2} \int d^{2} r d^{2} r^{\prime} v_{x}(\mathbf{r}) v_{x}\left(\mathbf{r}^{\prime}\right) \\
& \times G_{\mathbf{r}, \mathbf{r}^{\prime}}^{-}\left(E_{F}\right) G_{\mathbf{r}^{\prime}, \mathbf{r}}^{+}\left(E_{F}\right) .
\end{aligned}
$$

The Green function $G_{\mathbf{r}^{\prime}, \mathbf{r}}^{+}(E)$ is semiclassically approximated by a sum over classical trajectories $\gamma$ that start at the initial position $\mathbf{r}$ and end up at the final position $\mathbf{r}^{\prime}$ with energy $E$ given by ${ }^{30,31}$

$$
\begin{aligned}
G_{\mathbf{r}^{\prime}, \mathbf{r}}^{+}= & \sqrt{\frac{i}{2 \pi \hbar^{3}}} \sum_{\gamma} \exp \left(-\frac{T_{\gamma}}{2 \tau_{e l}}\right)\left|D_{\gamma}\right|^{1 / 2} \\
& \times \exp \left[i \frac{S_{\gamma}}{\hbar}\left(\mathbf{r}^{\prime}, \mathbf{r}\right)-i \frac{\pi}{2} \eta_{\gamma}\right] .
\end{aligned}
$$

$S_{\gamma}\left(\mathbf{r}^{\prime}, \mathbf{r}\right)=\int_{\gamma} \mathbf{p} \cdot d \mathbf{r}$ is the classical action of the trajectory $\gamma$ with the canonical momentum $\mathbf{p}, \eta_{\gamma}$ is the number of conjugate points along $\gamma, T_{\gamma}$ is the traversal time of the trajectory, and the first exponential is the weak disorder factor. The amplitude factor $D_{\gamma}$ involves second derivatives of $S_{\gamma}$. It is convenient to express it in terms of local coordinates $\mathbf{r}=(z, y)$, with coordinate $z$ along the orbit and $y$ perpendicular to it. Then

$$
D_{\gamma}=-\frac{1}{v v^{\prime}} \frac{\partial^{2} S_{\gamma}}{\partial y \partial y^{\prime}},
$$

where $v$ and $v^{\prime}$ are the velocities at $z$ and $z^{\prime}$. Inserting Eq. (3) into Eq. (2) one finds that the longitudinal conductivity is given by a double sum over oscillatory terms. Due to the fact that $G^{+}(E)$ and $G^{-}(E)$ are complex conjugates, $G_{\mathbf{r}, \mathbf{r}^{\prime}}^{-}(E)=\left[G_{\mathbf{r}^{\prime}, \mathbf{r}}^{+}(E)\right]^{*}$, the phase of the oscillatory terms contains the action difference of two trajectories $\left(\gamma_{1}\right.$ and $\left.\gamma_{2}\right)$. The conductivity is then split into two parts, a nonfluctuating one from the diagonal terms $\left(\gamma_{1}=\gamma_{2}=\gamma\right)$ and a fluctuating one from the nondiagonal terms (with $\gamma_{1} \neq \gamma_{2}$ ), that is

$$
\sigma_{x x} \approx \overline{\sigma_{x x}}+\sigma_{x x}^{f l} .
$$

The mean conductivity $\overline{\sigma_{x x}}$,

$$
\overline{\sigma_{x x}}=\frac{e^{2}}{h^{2} V} \int d^{2} r d^{2} r^{\prime} \sum_{\gamma\left(\mathbf{r}, \mathbf{r}^{\prime}\right)} v_{x}(\mathbf{r}) v_{x}\left(\mathbf{r}^{\prime}\right) D_{\gamma} \exp \left(-T_{\gamma} / \tau_{e l}\right),
$$

can be transformed into a familiar form as a phase-space average (denoted by $\langle\cdot\rangle_{\mathbf{r}, \mathbf{p}}$ ) where it is recognized as the Drude conductivity ${ }^{18,19}$

$$
\overline{\sigma_{x x}}=e^{2} \rho\left(E_{F}\right) \int_{0}^{\infty} d t\left\langle v_{x}(0) v_{x}(t)\right\rangle_{\mathbf{r}, \mathbf{p}} \exp \left(-t / \tau_{e l}\right),
$$

where $\rho\left(E_{F}\right)=m / 2 \pi \hbar^{2}$ is the density of states per unit area at the Fermi energy in two-dimensional systems.

The fluctuating part $\sigma_{x x}^{f l}$ is a quantum correction to the Drude conductivity and is given by the semiclassical formula

$$
\begin{aligned}
\sigma_{x x}^{f l}= & \frac{e^{2}}{h^{2} V} \sum_{\gamma_{1}, \gamma_{2}} \int d^{2} r d^{2} r^{\prime}\left(v_{1}\right)_{x}\left(v_{2}^{\prime}\right)_{x} \sqrt{D_{\gamma_{1}} D_{\gamma_{2}}} \\
& \times \exp \left(-\frac{T_{\gamma_{1}, \gamma_{2}}}{2 \tau_{e l}}\right) \\
& \times \exp \left(\frac{i}{\hbar} S_{\gamma_{1}, \gamma_{2}}\left(\mathbf{r}, \mathbf{r}^{\prime} ; E_{F}\right)-\frac{i \pi}{2} \eta_{\gamma_{1}, \gamma_{2}}\right),
\end{aligned}
$$

where $T_{\gamma_{1}, \gamma_{2}}=\left(T_{\gamma_{1}}+T_{\gamma_{2}}\right) ; S_{\gamma_{1}, \gamma_{2}}=S_{\gamma_{1}}-S_{\gamma_{2}}$, and $\eta_{\gamma_{1}, \gamma_{2}}=\eta_{\gamma_{1}}$ $-\eta_{\gamma_{2}}$. A correct evaluation of these integrals requires detailed knowledge of classical phase space structures as will become clear as we proceed.

The main contribution to the integrals comes from the stationary points where

$$
\begin{gathered}
\nabla S_{\gamma_{1}, \gamma_{2}}\left(\mathbf{r}^{\prime}, \mathbf{r} ; E_{F}\right)=\mathbf{p}_{2}-\mathbf{p}_{1}=0, \\
\nabla^{\prime} S_{\gamma_{1}, \gamma_{2}}\left(\mathbf{r}^{\prime}, \mathbf{r} ; E_{F}\right)=\mathbf{p}^{\prime}{ }_{1}-\mathbf{p}_{2}^{\prime}=0 .
\end{gathered}
$$

Here $\mathbf{p}_{i}$ and $\mathbf{p}^{\prime}{ }_{i}$ denote the initial and final momenta of trajectory $\gamma_{i}$. The two trajectories $\gamma_{1}$ and $\gamma_{2}$ must hence have the same initial and final momenta in addition to having the same initial and final positions. One way of satisfying these conditions with $\gamma_{1}$ and $\gamma_{2}$ not being identical is that both trajectories are part of a primitive periodic orbit $\gamma$. In fact, for each periodic orbit $\gamma$ there is an infinite set of pairs that satisfy the stationary-phase conditions. They differ only by 
the number of times they wind around $\gamma$ and their action difference is a multiple of the action of the primitive periodic orbit $S_{\gamma_{1}}-S_{\gamma_{2}}=r S_{\gamma}$.

The evaluation of Eq. (8) is closely related to performing the trace in the derivation of Gutzwiller's trace formula. The integration is done in the local coordinates $\mathbf{r}=(z, y)$ with $d^{2} r d^{2} r^{\prime}=d z d z^{\prime} d y d y^{\prime}$. In the case of isolated periodic orbits the integrals over $y$ and $y^{\prime}$ are evaluated by the stationary phase method while the integrals over $z$ and $z^{\prime}$ are performed exactly. ${ }^{18,19}$ This results in

$$
\begin{aligned}
\sigma_{x x}^{f l}= & \frac{2 e^{2}}{h V} \sum_{\gamma} \sum_{r=1}^{\infty} \exp \left(\frac{-r T_{\gamma}}{2 \tau_{e l}}\right) C_{\gamma}\left(v_{x}, v_{x}\right) \\
& \times \frac{\cos \left[r S_{\gamma}\left(E_{F}\right) / \hbar-\pi r \mu_{\gamma} / 2\right]}{\left|\operatorname{det}\left(M_{\gamma}-1\right)\right|^{1 / 2}},
\end{aligned}
$$

where $C_{\gamma}\left(v_{x}, v_{x}\right)$ is a correlation function of the longitudinal components of the velocity along the orbit $\gamma$

$$
C_{\gamma}\left(v_{x}, v_{x}\right)=\int_{0}^{T_{\gamma}} d t \int_{0}^{\infty} d t^{\prime} v_{x}(t) v_{x}\left(t+t^{\prime}\right) e^{-t^{\prime} / \tau_{e l}}
$$

Formula (10) applies to the fully chaotic case where all orbits are unstable. In a lattice there are many copies of an orbit, so when counting different orbits one has to multiply each one with a degeneracy factor, depending on the geometry. Finite temperature and spin effects lead to additional factors of $\left(\pi r T_{\gamma} / \hbar \beta\right) / \sinh \left(\pi r T_{\gamma} / \hbar \beta\right)$ and $2 \cos \left(r T_{\gamma} \mu_{B} B / \hbar\right)$, respectively, where $\mu_{B}=e \hbar / 2 m_{e}$ is the Bohr magneton. There are similar formulas for the Hall conductivity $\sigma_{x y} .3,18,19$

Close to a bifurcation the saddle-point approximation leading to Eq. (10) breaks down because the saddle point is not isolated. There are nearby saddle points from the other periodic orbits that participate in the bifurcation. This occurs when, by changing the energy or parameters of the system, the eigenvalues of $M^{r} \rightarrow 1$ and Eq. (10) diverges. In order to obtain the correct semiclassical contribution one has to integrate over the neighboring saddle points as well. This results in transitional or uniform approximations for the bifurcating orbits.

Bifurcations occur in specific forms that depend on the repetition number $r$ for which det $M^{r}=1$. They are characterized by normal forms which describe the characteristic motion of trajectories in the vicinity of a periodic orbit. ${ }^{34}$ Before describing the normal forms we transform the integral in Eq. (8) into a form that is more appropriate for treating bifurcations.

One of the integrals over $y$ and $y^{\prime}$ is done in stationary phase approximation, and the other is transformed to an integral over normal form coordinates. ${ }^{35,36}$ Afterwards the integral over the $z$ and $z^{\prime}$ coordinates can be performed. The resulting contribution from the $r$ th repetition of a bifurcating orbit $\gamma$ is

$$
\frac{e^{2} \exp \left(-\frac{r T_{\gamma}}{2 \tau_{e l}}\right)}{2 \pi^{2} V} C_{\gamma}\left(v_{x}, v_{x}\right) \Re\left[\exp \left(\frac{i}{\hbar} r S_{0}-i \frac{\pi}{2} \nu_{r}\right) \mathcal{G}\left(E_{F}\right)\right],
$$

where

$$
\mathcal{G}\left(E_{F}\right)=\frac{1}{\hbar^{2}} \int d Q^{\prime} d P \exp \left(\frac{i}{\hbar} \Phi\left(Q^{\prime}, P\right)\right) .
$$

Here $S_{0}$ is the action of the periodic orbit at the center of the Poincare surface, and $\nu_{r}$ is the number of conjugate points along $r$ repetitions of this orbit. $\Phi\left(Q^{\prime} P\right)$ is a generating function for the Poincare map in normal form coordinates from $(Q, P)$ to $\left(Q^{\prime}, P^{\prime}\right)$. At the position of a periodic point

$$
\frac{\partial \Phi}{\partial Q^{\prime}}=P^{\prime}-P=0, \quad \frac{\partial \Phi}{\partial P}=Q-Q^{\prime}=0,
$$

and $\Phi$ is stationary.

Equation (12) is a transitional approximation for the bifurcating orbits. It is correct when the orbits are close to a bifurcation, where its semiclassical effect is largest. ${ }^{35}$ If, due to a change of parameters, the orbits move further apart, it reduces to a sum of single terms as in Eq. (10) where, however, the amplitudes for the neighboring orbits are inaccurate. The transitional approximation is sufficient for our purpose. A uniform approximation which has the correct single orbit limit can be obtained by including the correlation function $C_{\gamma}\left(v_{x}, v_{x}\right)$ into the integral (where it is evaluated along neighboring trajectories) and including also the $Q^{\prime}$ and $P$ dependence of the preexponential factor that comes from the determinants $D .{ }^{36,37}$ In Eq. (12) this factor has been evaluated at the central orbit and has the value one.

Let us look at an example of a pitchfork bifurcation with normal form

$$
\Phi(Q, P)=\frac{P^{2}}{2}+\varepsilon Q^{2}+c Q^{4},
$$

where $c>0$ and the primes have been dropped for convenience. For $\varepsilon>0$ there is only one solution of $0=\partial \Phi / \partial Q$ $=\partial \Phi / \partial P$ at $(0,0)$ corresponding to the central periodic orbit. If, by changing parameters, $\varepsilon$ goes through zero, two new solutions appear which are located symmetrically about $Q=0$. In the generic situation (in systems without symmetries) this is a period doubling bifurcation. It occurs if the lowest repetition number $r$ for which $\operatorname{det}\left(M^{r}-1\right)=0$ is $r=2$. At the bifurcation one new orbit of double the period arises which intersects the Poincaré section twice.

The integral (13) can be evaluated in closed form for the normal form (15) and is given by a sum of two Bessel functions with index $\frac{1}{4}$ and $-\frac{1}{4}$. At the bifurcation, $\varepsilon=0$, it has the simple form

$$
\mathcal{G}(E)=\sqrt{\frac{\pi}{8}} \frac{\Gamma(1 / 4) \exp (3 \pi i / 4)}{\hbar^{5 / 4} c^{1 / 4}} .
$$

The exponent of $\hbar$ in the denominator, $\beta=5 / 4$, shows that the bifurcation term is by a factor $\hbar^{-1 / 4}$ stronger than that of 
TABLE I. The relevant parts of the normal forms for bifurcations of period- $r$ orbits with codimension $K=2$.

\begin{tabular}{cc}
\hline \hline$r$ & $\Phi_{r, 2}$ \\
\hline 1 & $P^{2}+x_{1} Q+x_{2} Q^{2}+Q^{4}$ \\
2 & $P^{2}+x_{1} Q^{2}+x_{2} Q^{4}+Q^{6}$ \\
3 & $\left(P^{2}+Q^{2}\right)^{2}+x_{1}\left(P^{2}+Q^{2}\right)+x_{2} \operatorname{Re}\left[(P+i Q)^{3}\right]$ \\
4 & $P^{2} Q^{2}+x_{1}\left(P^{2}+Q^{2}\right)+x_{2}\left(P^{2}-Q^{2}\right)^{2}$ \\
5 & $\operatorname{Re}\left[(P+i Q)^{5}\right]+x_{1}\left(P^{2}+Q^{2}\right)+x_{2}\left(P^{2}+Q^{2}\right)^{2}$ \\
$\geqslant 6$ & $\left(P^{2}+Q^{2}\right)^{3}+x_{1}\left(P^{2}+Q^{2}\right)+x_{2}\left(P^{2}+Q^{2}\right)^{2}$ \\
\hline
\end{tabular}

a single orbit. The contribution of pitchfork and tangent bifurcations to the conductivity were evaluated in Refs. 3 and 23.

We note that the constant $c$ in the normal form that appears in Eq. (16) can be obtained by following the actions and stabilities of the orbits through the bifurcations. It follows from $^{37}$

$$
\operatorname{Tr} M_{0}-\operatorname{Tr} M_{1} \sim-6 \varepsilon, \quad S_{0}-S_{1} \sim \frac{\varepsilon^{2}}{4 c} .
$$

Hence one finds

$$
c=\lim _{\varepsilon \rightarrow 0} \frac{\left(\operatorname{Tr} M_{0}-\operatorname{Tr} M_{1}\right)^{2}}{144\left(S_{0}-S_{1}\right)} .
$$

There is a second exponent, $\gamma$, which characterizes the semiclassical importance of bifurcations for spectral and transport properties. It specifies the region in parameter space ( $\varepsilon$ in the example) in which the contribution of the bifurcation is strongest. Both $\beta$ and $\gamma$ can be obtained from the normal form by a scaling argument. We perform a scaling of $Q, P$, and $\varepsilon$ such that the integral in Eq. (13) takes the form

$$
\mathcal{G}\left(E_{F}, \varepsilon, \hbar\right)=\frac{1}{\hbar^{\beta}} \mathcal{G}\left(E_{F}, \frac{\varepsilon}{\hbar^{\gamma}}, 1\right) .
$$

In the example of the pitchfork bifurcation the argument of the exponent is made $\hbar$-independent by changing the integration variables and a subsequent scaling of $\varepsilon$.

$$
P=\hbar^{1 / 2} \widetilde{P}, \quad Q=\hbar^{1 / 4} \widetilde{Q}, \quad \varepsilon=\hbar^{1 / 2} \widetilde{\varepsilon} .
$$

As a result $\beta=2-1 / 2-1 / 4=5 / 4$, as before, and $\gamma=1 / 2$.

Different bifurcations have different exponents $\beta$ and $\gamma$ and, depending on the quantities that one considers, they are of different importance. We will consider in the following statistical properties of the conductivity. It will be shown that they are dominated by certain kinds of bifurcations, leading to universal properties in the regime of mixed classical dynamics. Before we do that we give an overview of classifications of bifurcations that are known presently.

The generic bifurcations of codimension $K=1$ are those that occur if one parameter of the system is varied. They have been classified by Meyer and Bruno. ${ }^{32,33}$ A list of the normal forms can be obtained from Table I by setting $x_{2}=1$ (except for $r=1$ where it is $P^{2}+x_{1} Q+Q^{3}$ ). These normal
TABLE II. Exponents $\beta_{K, r}$ and $\gamma_{K, r}$ for generic bifurcations with codimension $K=1$ or $K=2$.

\begin{tabular}{ccccc}
\hline \hline$r$ & $\beta_{1, r}$ & $\gamma_{1, r}$ & $\beta_{2, r}$ & $\gamma_{2, r}$ \\
\hline 1 & $\frac{7}{6}$ & $\frac{2}{3}$ & $\frac{5}{4}$ & $\frac{5}{4}$ \\
2 & $\frac{5}{4}$ & $\frac{1}{2}$ & $\frac{4}{3}$ & 1 \\
3 & $\frac{4}{3}$ & $\frac{1}{3}$ & $\frac{3}{2}$ & $\frac{3}{4}$ \\
4 & $\frac{3}{2}$ & $\frac{1}{2}$ & $\frac{3}{2}$ & $\frac{1}{2}$ \\
5 & $\frac{3}{2}$ & $\frac{1}{2}$ & $\frac{8}{5}$ & $\frac{4}{5}$ \\
26 & $\frac{3}{2}$ & $\frac{1}{2}$ & $\frac{5}{3}$ & 1
\end{tabular}

forms are simplified versions in which all constants and terms that are irrelevant for the determination of $\beta$ and $\gamma$ have been removed. The scaling proceedure of Eq. (19) yields the exponents $\beta_{r}$ and $\gamma_{r}$ that are given in Table II.

If more parameters than one are varied then other bifurcations occur in which more complicated configurations of periodic orbits coalesce. For example, by varying a second parameter one can make certain codimension $K=1$ bifurcations occur at the same instance. The codimension $K$ corresponds to the number of parameters that are varied. Generic bifurcations of codimension $K=2$ have been classified by Schomerus, ${ }^{38}$ and the normal forms are given in Table I. For each parameter $x_{j}$ one has an exponent $\sigma_{j}$ that specifies the range over which the bifurcation is important, $\Delta x_{j} \sim \hbar^{\sigma_{j}}$. The total volume in $K$-dimensional parameter space then scales as $\hbar^{\gamma}$ where

$$
\gamma=\sum_{j=1}^{K} \sigma_{j} .
$$

The exponents $\beta_{K, r}$ and $\gamma_{K, r}$ for bifurcations of codimension $K=2$ are also listed in Table II.

Although bifurcations of higher codimension do not occur, in general, when only one parameter is varied, they still affect semiclassical approximations because of their finite extension in parameter space. This is one of the main reasons why the semiclassical analysis of mixed systems is so intricate.

For bifurcations of codimension $K \geqslant 3$ there is no complete classification. Only partial results exist. Our main interest lies in bifurcations of arbitrary codimension $K$ with repetition number $r \geqslant 2 K+2$. These have the (simplified) normal form

$$
\Phi_{r, K}(Q, P)=I^{K+1}+\sum_{n=1}^{K} x_{n} I^{n}+O\left(I^{K+2}\right),
$$

where $I=Q^{2}+P^{2}$. Expressing $\Phi$ in terms of $Q$ and $P$, we find 


$$
\begin{gathered}
\beta_{K, r}=2-\frac{2}{2(K+1)}=\frac{2 K+1}{K+1}, \\
\sigma_{K, r, n}=1-\frac{n}{K+1} \Rightarrow \gamma_{K, r}=\sum_{n=1}^{K} \sigma_{K, r, n}=\frac{K}{2} .
\end{gathered}
$$

It will be shown that these bifurcations are the most important for determining conductance fluctuations in the next section.

\section{MOMENTS OF THE CONDUCTIVITY FLUCTUATIONS}

We estimate the semiclassical size of the conductivity fluctuations $\sigma_{x x}^{f l}$ by evaluating the $\hbar$-dependence of the moments

$$
M_{2 m}(\hbar)=\left\langle\left[\sigma_{x x}^{f l}(E, \hbar)\right]^{2 m}\right\rangle,
$$

where $\langle\cdot\rangle$ denotes a local average over one or more parameters of the Hamiltonian, for example, Fermi energy, magnetic field, or system parameters. In this section we assume that very long orbits do contribute to the longitudinal conductivity. The consequence of the damping of the contributions of long orbits due to disorder and inelastic processes is discussed in the next section. The central point now is to replace the average by an average over the parameters in the normal forms

$$
M_{2 m, r, K}(\hbar) \equiv B \int d^{K} \mathbf{x}\left[\sigma_{r, K}^{f l}(\mathbf{x}, \hbar)\right]^{2 m}
$$

where $B$ is a normalization constant. The scaling procedure in Eq. (19) yields that each bifurcation, labeled by $r$ and $K$, contributes a term that scales as $1 / \hbar^{2 m \beta_{K, r}-\gamma_{K, r}}$. With the $\hbar$ dependence thus extracted, these contributions can now be compared for different bifurcations. The bifurcation that wins the competition is that for which the $\hbar$ exponent in the denominator is largest, and it determines that rate at which the $\mathbf{x}$-averaged moments diverge in the semiclassical limit. That is

$$
M_{2 m}(\hbar) \sim \hbar^{-\nu_{m}} \quad \text { as } \hbar \rightarrow 0,
$$

where

$$
\nu_{m}=\max _{K, r}\left(2 m \beta_{K, r}-\gamma_{K, r}\right) .
$$

The exponents $\nu_{m}$ are universal numbers that are determined by studying the hierarchy of bifurcations. Similar universal exponents have also been found for moments of the fluctuating parts of the spectral counting function and wave functions. ${ }^{25-27}$ They have been named "twinkling exponents," in analogy to the exponents that control the intensity of twinkling starlight.

\begin{tabular}{|c|c|c|c|c|}
\hline$r$ & $\nu_{1,1, r}$ & $\nu_{2,1, r}$ & $\nu_{1,2, r}$ & $\nu_{2,2, r}$ \\
\hline \multirow{2}{*}{1} & 5 & 12 & 5 & 15 \\
\hline & $\overline{3}$ & $\overline{3}$ & $\overline{4}$ & $\overline{4}$ \\
\hline \multirow[t]{2}{*}{2} & 2 & 9 & 5 & 13 \\
\hline & & $\overline{2}$ & $\overline{3}$ & 3 \\
\hline \multirow[t]{2}{*}{3} & 7 & 5 & 9 & 21 \\
\hline & $\overline{3}$ & & $\overline{4}$ & $\overline{4}$ \\
\hline \multirow[t]{2}{*}{4} & $\underline{5}$ & 11 & 5 & 11 \\
\hline & $\overline{2}$ & $\overline{2}$ & $\overline{2}$ & $\overline{2}$ \\
\hline \multirow[t]{2}{*}{5} & $\underline{5}$ & 11 & 12 & 28 \\
\hline & $\overline{2}$ & $\overline{2}$ & $\overline{5}$ & $\overline{5}$ \\
\hline \multirow{2}{*}{$\geqslant 6$} & 5 & 11 & 7 & 17 \\
\hline & $\overline{2}$ & 2 & $\overline{3}$ & 3 \\
\hline
\end{tabular}

For the generic bifurcations with $K=1$ and $K=2$ the exponents $\nu_{m, K, r}=2 m \beta_{K, r}-\gamma_{K, r}$ are listed in Table III. For generic bifurcations with $K>2$ and $r \geqslant 2 K+2$ the exponents $\nu_{m, K, r}$ follow from Eq. (23) and are given by
TABLE III. Values of $\nu_{m, K, r}=2 m \beta_{K, r}-\gamma_{K, r}$.

$$
\nu_{m, K, r}=2 m \frac{2 K+1}{K+1}-\frac{K}{2},
$$

which are independent of $r$. Although the normal forms for $K>3$ and $1 \leqslant r<K$ have not been classified completely, it has been argued in Refs. 26 and 27 that these bifurcations cannot contribute to the twinkling exponents since they have a counterpart with $\tilde{K}<K$ and $\tilde{r}>2 \tilde{K}+2$ with the property that $\nu_{m, \tilde{K}, \tilde{r}}>\nu_{m, K, r}$. The same argument can be applied in the present case, and hence the maxima of the exponents given in Eq. (28) represent the maxima with respect to all generic bifurcations and are universal. They can be written as

$$
\nu_{m}=4 m-\min _{K}\left(\frac{2 m}{K+1}+\frac{K}{2}\right) .
$$

The exponents $\nu_{m}$ are given in Table IV. If $m$ is a square then $\nu_{m}=4 m-2 \sqrt{m}+1 / 2$. If not, the maximum value is attained at one or both of the two integer values of $K$ that are closest to $2 \sqrt{m}-1$. It is clear from the moments that the conductivity fluctuations do not have a Gaussian distribution.

Another quantity explored in the literature concerns the magnetic fingerprint in antidot lattices incorporated in the autocorrelation function of the conductivity. ${ }^{39}$ Consider

$$
\mathcal{F}(\Delta x)=\left\langle\sigma_{x x}^{f l}(x+\Delta x) \times \sigma_{x x}^{f l}(x)\right\rangle_{x, y},
$$

where $\sigma_{x x}^{f l}$ is written as a sum over classical orbits and $x$ is a parameter of the system, e.g., the magnetic field. The average $\langle\cdot\rangle_{x, y}$ might be over other parameters as well. At $\Delta x=0$ this is identical to the second moment $M_{2}$. The bifurcations then determine the characteristic length scale over which the correlations decay as $\Delta x$ is increased. In the generic situation all

TABLE IV. Exponents $\nu_{m}$ and codimensions $K$ of the dominating bifurcations for generic two-dimensional systems.

\begin{tabular}{ccccccccc}
\hline \hline$m$ & 1 & 2 & 3 & 4 & 5 & 6 & 7 & 8 \\
\hline$\nu_{m}$ & $2 \frac{1}{2}$ & $5 \frac{2}{3}$ & 9 & $12 \frac{1}{2}$ & 16 & $19 \frac{3}{5}$ & $23 \frac{1}{5}$ & $26 \frac{5}{6}$ \\
$K$ & 1 & 2 & 2,3 & 3 & 3,4 & 4 & 4 & 5 \\
\hline \hline
\end{tabular}


parameters $x_{n}$ in the normal form are affected when the physical parameter $x$ is changed. Hence the correlation length scales as $\hbar^{\sigma}$ where $\sigma$ is the minimum of the $\sigma_{i}$. For generic bifurcations with $r \geqslant 2 K+2$ this yields $\sigma=1 /(K+1)$.

\section{DISCUSSION}

The semiclassical analysis of the conductivity fluctuations leads to a power-law dependence of the moments $M_{2 m}$ $\sim 1 / \hbar^{\nu_{m}}$, with fractional exponents $\nu_{m}$. In situations where arbitrarily long periodic orbits contribute to the conductivity these exponents are universal numbers that are obtained from the competition between different bifurcation. The analysis was done in terms of $\hbar$. If a different semiclassical variable is used instead of $\hbar$, e.g., the Fermi wave length, then the exponents have to be adjusted depending on how the classical action scales with the semiclassical parameter.

In experiments most of the long orbits are suppressed and the conductivity is determined by a relatively small number of periodic orbits. One cannot expect then, in general, to see the universal exponents in particular for the higher moments, because they originate from high repetitions of periodic orbits. Instead the exponents will be determined by the competition within the much smaller class of those bifurcations that affect the relevant periodic orbits. The dominating exponent can then be found by comparing the corresponding exponents $\nu_{m, K, r}$ in Table III (assuming that higher codimen- sions are not important). Nevertheless, also in this case the conductivity fluctuations would be non-Gaussian and the moments would have a power-law dependence with fractional exponents in clear contrast to the chaotic case with Gaussian fluctuations and $\nu_{m}=2 \mathrm{~m}$. Also the length scale of the decay of correlations is larger if the classical dynamics is mixed, and is of order $\hbar^{\sigma}$ with $\sigma<1$.

There is, however, one property of antidot lattices that might make it possible to observe the universal exponents for the lower moments, and that is their high symmetry. In systems with discrete symmetries there are also nongeneric bifurcations which typically have the same normal form as generic bifurcations, but at lower repetition numbers. For example, the pitchfork bifurcation Eq. (15) can occur at repetition number $r=1$ where two new orbits of the same length appear instead of one orbit of double the length. ${ }^{40}$ Similarly, if the antidot lattice is, e.g., invariant under rotations of $\pi / 3$ then a bifurcation that is generic for $r=6$ can occur at the first repetition of a periodic orbit. ${ }^{41}$ This might make it feasible to observe the universal regime that in nonsymmetric systems is restricted to very long orbits.

\section{ACKNOWLEDGMENTS}

We are grateful to the Royal Society for funding this work. J.P.K. is supported by an EPSRC Senior Research Fellowship. S.D.P. thanks FAPERGS for partial support.
${ }^{1}$ Proceedings of the 1994 Les Houches Summer School on Mesoscopic Quantum Physics, edited by E. Akkermans, G. Montambaux, J.-L. Pichard, and J. Zinn-Justin (North-Holland, Amsterdam, 1995).

${ }^{2}$ Special issue, Chaos, Solitons Fractals 8, 971 (1997).

${ }^{3}$ K. Nakamura and T. Harayama, Quantum Chaos and Quantum Dots (Oxford University Press, Oxford, 2004).

${ }^{4}$ R. A. Jalabert, Proceedings of the International School of Physics “Enrico Fermi," Course CXLIII (IOS Press, Amsterdam, 2000), pp. 145-222.

${ }^{5}$ K. Richter, Semiclassical Theory of Mesoscopic Quantum Systems (Springer, Berlin, 2000).

${ }^{6}$ R. Blümel and U. Smilansky, Phys. Rev. Lett. 60, 477 (1988); Physica D 36, 111 (1989); E. Doron, U. Smilansky, and A. Frenkel, ibid. 50, 367 (1991).

${ }^{7}$ R. A. Jalabert, H. U. Baranger, and A. D. Stone, Phys. Rev. Lett. 65, 2442 (1990); H. U. Baranger, R. A. Jalabert, and A. D. Stone, Chaos 3, 665 (1993).

${ }^{8}$ C. M. Marcus, A. J. Rimberg, R. M. Westervelt, P. F. Hopkins, and A. C. Gossard, Phys. Rev. Lett. 69, 506 (1992).

${ }^{9}$ H. U. Baranger, R. A. Jalabert, and A. D. Stone, Phys. Rev. Lett. 70, 3876 (1993).

${ }^{10}$ M. W. Keller, O. Millo, A. Mittal, D. E. Prober, and R. N. Snacks, Surf. Sci. 305, 501 (1994); M. W. Keller, A. Mittal, J. W. Sleight, R. G. Wheeler, D. E. Prober, R. N. Sacks, and H. Shtrikmann, Phys. Rev. B 53, R1693 (1996).

${ }^{11}$ A. M. Chang, H. U. Baranger, L. N. Pfeiffer, and K. W. West, Phys. Rev. Lett. 73, 2111 (1994).
${ }^{12}$ P. Pichaureau and R. A. Jalabert, Eur. Phys. J. B 9, 299 (1999).

${ }^{13}$ L. Wirtz, J.-Z. Tang, and J. Burgdörfer, Phys. Rev. B 56, 7589 (1997).

${ }^{14}$ K. Richter, D. Ullmo, and R. A. Jalabert, Phys. Rep. 276, 1 (1996).

${ }^{15}$ D. Weiss, M. L. Roukes, A. Menschig, P. Grambow, K. von Klitzing, and G. Weimann, Phys. Rev. Lett. 66, 2790 (1991).

${ }^{16}$ R. Fleischmann, T. Geisel, and R. Ketzmerick, Phys. Rev. Lett. 68, 1367 (1992).

${ }^{17}$ D. Weiss, K. Richter, A. Menschig, R. Bergmann, H. Schweizer, K. von Klitzing, and G. Weimann, Phys. Rev. Lett. 70, 4118 (1993).

${ }^{18}$ G. Hackenbroich and F. von Oppen, Europhys. Lett. 29, 151 (1995); Z. Phys. B: Condens. Matter 97, 157 (1995).

${ }^{19}$ K. Richter, Europhys. Lett. 29, 7 (1995).

${ }^{20}$ R. Ketzmerick, Phys. Rev. B 54, 10841 (1996).

${ }^{21}$ A. S. Sachrajda, R. Ketzmerick, C. Gould, Y. Feng, P. J. Kelly, A. Delage, and Z. Wasilewski, Phys. Rev. Lett. 80, 1948 (1998); R. P. Taylor, A. P. Micolich, T. M. Fromhold, and R. Newbury, ibid. 83, 1074 (1999); A. S. Sachrajda and R. Ketzmerick, ibid. 83, 1075 (1999).

${ }^{22}$ F. Nihey, S. W. Hwang, and K. Nakamura, Phys. Rev. B 51, 4649 (1995).

${ }^{23}$ J. Ma and K. Nakamura, Phys. Rev. B 62, 13552 (2000).

${ }^{24}$ M. V. Berry, J. P. Keating, and S. D. Prado, J. Phys. A 31, L245 (1998).

${ }^{25}$ M. V. Berry, Proceedings of the International School of Physics “Enrico Fermi,” Course CXLIII (IOS Press, Amsterdam, 2000), 
pp. $45-63$.

${ }^{26}$ M. V. Berry, J. P. Keating, and H. Schomerus, Proc. R. Soc. London, Ser. A 456, 1659 (2000).

${ }^{27}$ J. P. Keating and S. D. Prado, Proc. R. Soc. London, Ser. A 457, 1855 (2001).

${ }^{28}$ M. Wilkinson, J. Phys. A 20, 2415 (1987).

${ }^{29}$ C. Středa, J. Phys. C 15, L717 (1982).

${ }^{30}$ M. C. Gutzwiller, J. Math. Phys. 12, 343 (1971).

${ }^{31}$ M. C. Gutzwiller, Chaos in Classical and Quantum Mechanics (Springer, Berlin, 1990).

${ }^{32}$ K. R. Meyer, Trans. Am. Math. Soc. 149, 95 (1970).

${ }^{33}$ A. D. Brjuno, Math. USSR. Sb. 12, 271 (1970).

${ }^{34}$ A. M. Ozorio de Almeida, Hamiltonian Systems: Chaos and
Quantization (Cambridge University Press, Cambridge, England, 1988).

${ }^{35}$ A. M. Ozorio de Almeida and J. H. Hannay, J. Phys. A 20, 5837 (1987).

${ }^{36}$ M. Sieber, J. Phys. A 29, 4715 (1996).

${ }^{37}$ H. Schomerus and M. Sieber, J. Phys. A 30, 4537 (1997); M. Sieber and H. Schomerus, ibid. 31, 165 (1998).

${ }^{38}$ H. Schomerus, J. Phys. A 31, 4167 (1998).

${ }^{39}$ T. Nakanishi and T. Ando, Phys. Rev. B 54, 8021 (1996).

${ }^{40}$ M. A. M. Aguiar, C. P. Malta, M. Baranger, and K. T. R. Davies, Ann. Phys. (Paris) 180, 167 (1987).

${ }^{41}$ H. Then, Diploma thesis, University of Ulm, 1999. 